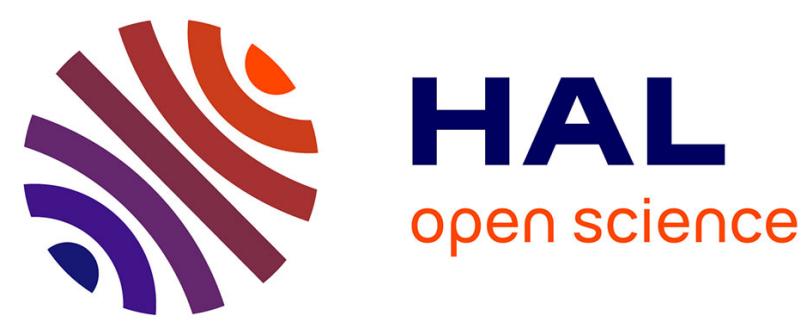

\title{
L'occupation du site et son contexte environnemental
}

Olivier Buchsenschutz, Hervé Richard, Jean-Paul Guillaumet, Vincent

Guichard, Patrice Beck, Walter Berry, Christine Canat, Katherine Gruel, Josef Laszlovszky, Benjamin Saint-Jean-Vitus, et al.

\section{To cite this version:}

Olivier Buchsenschutz, Hervé Richard, Jean-Paul Guillaumet, Vincent Guichard, Patrice Beck, et al.. L'occupation du site et son contexte environnemental. Gallia - Archéologie de la France antique, 1998, L'oppidum de Bibracte, 55, pp.8-18. 10.3406/galia.1998.3258 . hal-01914609

\author{
HAL Id: hal-01914609 \\ https://hal.science/hal-01914609
}

Submitted on 11 Mar 2020

HAL is a multi-disciplinary open access archive for the deposit and dissemination of scientific research documents, whether they are published or not. The documents may come from teaching and research institutions in France or abroad, or from public or private research centers.
L'archive ouverte pluridisciplinaire HAL, est destinée au dépôt et à la diffusion de documents scientifiques de niveau recherche, publiés ou non, émanant des établissements d'enseignement et de recherche français ou étrangers, des laboratoires publics ou privés.

\section{(ㅇ)(1) $\$$}

Distributed under a Creative Commons Attribution - NonCommercial - NoDerivatives| 4.0 
fices successifs, que les aménagements de la Fontaine Saint-Pierre se sont succédé de la fin de l'époque gauloise au XIX ${ }^{\mathrm{e}}$ s... L'étude conjointe des stratigraphies et des « ensembles clos" de mobilier livrés par les fouilles récentes permet enfin de subdiviser l'occupation antique du site en plusieurs horizons chronologiques qui s'échelonnent de la seconde moitié du II' $\mathrm{s}$. avant J.-C. au règne d'Auguste.

J.-P. G., P.-P. B.

\section{LA REPRISE DES FOUILLES DEPUIS 1984}

Depuis 1984, une douzaine d'équipes dirigées par des universitaires et des chercheurs a été amenée à fouiller au Mont Beuvray. Ces équipes interviennent généralement un mois chacune, ce qui représente environ de 3500 à 4500 journées ouvrées par an. Ces travaux de terrain sont intégrés dans le cursus des universités européennes participantes. De 1988 à 1993, le programme européen Erasmus, porté d'abord par l'université Paris-I (O. Buchsenschutz) puis par l'École du Louvre (A. Duval), a concrétisé cette coopération interuniversitaire pour la formation pratique des étudiants en archéologie.

Une équipe du CNRS (l'URA 33, aujourd'hui UMR 126) effectue en 1984 un premier sondage dans le rempart, à la Porte du Rebout, pour évaluer la complexité des fortifications. En 1985, un deuxième programme de fouilles est lancé sur les sanctuaires de la Chaume. À partir de 1986, l'opération est ouverte à d'autres équipes, tant françaises qu'étrangères.

Tout en achevant les programmes déjà lancés précédemment, il est décidé dès 1987 de concentrer les nouvelles équipes sur la fouille d'une zone d'habitat le long de l'axe montant de la Porte du Kebout à la Pâture du Couvent et au Parc aux Chevaux. L'équipe du CNRS précitée engage ainsi une fouille étendue sur la Pâture du Couvent, tandis que l'université de Lausanne (Suisse) entame en 1988 un sondage stratigraphique sur la domus PC 1. L'arrivée d'H. Richard, du laboratoire de Chronoécologie de Besançon (actuelle UMR 9946 du CNRS), entraîne l'ouverture d'un chantier en milieu humide, à la Fontaine Saint-Pierre, lié aux problématiques environnementales. Par ailleurs, les fouilles de la Chaume se poursuivent par l'exploration de l'espace contigu de la Terrasse. Les fouilles de la Pâture du Couvent, encore inachevées, ont pris une grande ampleur et deviennent le principal chantier, avec le dégagement d'une des voies principales de l'oppidum, ornée d'un bassin monumental, de deux îlots de constructions du I ${ }^{\text {er }}$ s. avant J.-C., ainsi que des ruines d'un couvent franciscain.

De 1992 à 1994, les différents travaux nécessaires à la construction du musée de Bibracte ont aussi entrainé de multiples surveillances et des fouilles de sauvetage assurées par l'équipe permanente du Centre archéologique du Mont Beuvray, notamment la mise au jour d'une des nécropoles de l'oppidum ( $c f$. infra, p. 43). Une petite équipe d'archéologues salariés a par ailleurs été chargée, en 1994 et 1995, de préciser la localisation de constructions fouillées au XIX' $\mathrm{x}$. sur le secteur central de l'oppidum. Quant aux prospections géophysiques, elles ont porté au total sur 8 ha et les relevés topographiques divers sur 11 ha environ. Parmi ces derniers, il faut mentionner tout particulièrement le positionnement par F. Schubert du rempart extérieur, découvert en 1985, sur un fond de plan photogrammétrique au 1/2500.

Parallèlement aux recherches de terrain, des chercheurs ont pris en charge l'étude globale de certains types de mobiliers archéologiques ou de documents. Une première partie de ces travaux a été consacrée à la documentation ancienne de Bulliot et Déchelette (notamment Laubenheimer, 1991 ; Guillaumet, 1996, qui font suite à Guillaumet, 1979 et 1984). Dans une deuxième étape, ces recherches ont pris en compte les données des fouilles récentes, qui ne sont devenues suffisantes pour justifier les premières synthèses qu'à partir du début des années 1990.

Onze ans après la reprise des recherches, les découvertes archéologiques ont considérablement modifié les caractères du site tels qu'ils avaient pu être définis par Bulliot et Déchelette.

J.-L. F., K. G.

\section{L'OCCUPATION DU SITE ET SON CONTEXTE ENVIRONNEMENTAL}

\section{L'ENVIRONNEMENT}

Dès le début du programme engagé sur l'oppidum de Bibracte, il a été clairement établi que les thèmes de recherches principaux devaient être replacés dans leurs 
Fig. 3 - Le Mont Benvray vu depuis le sud-est.

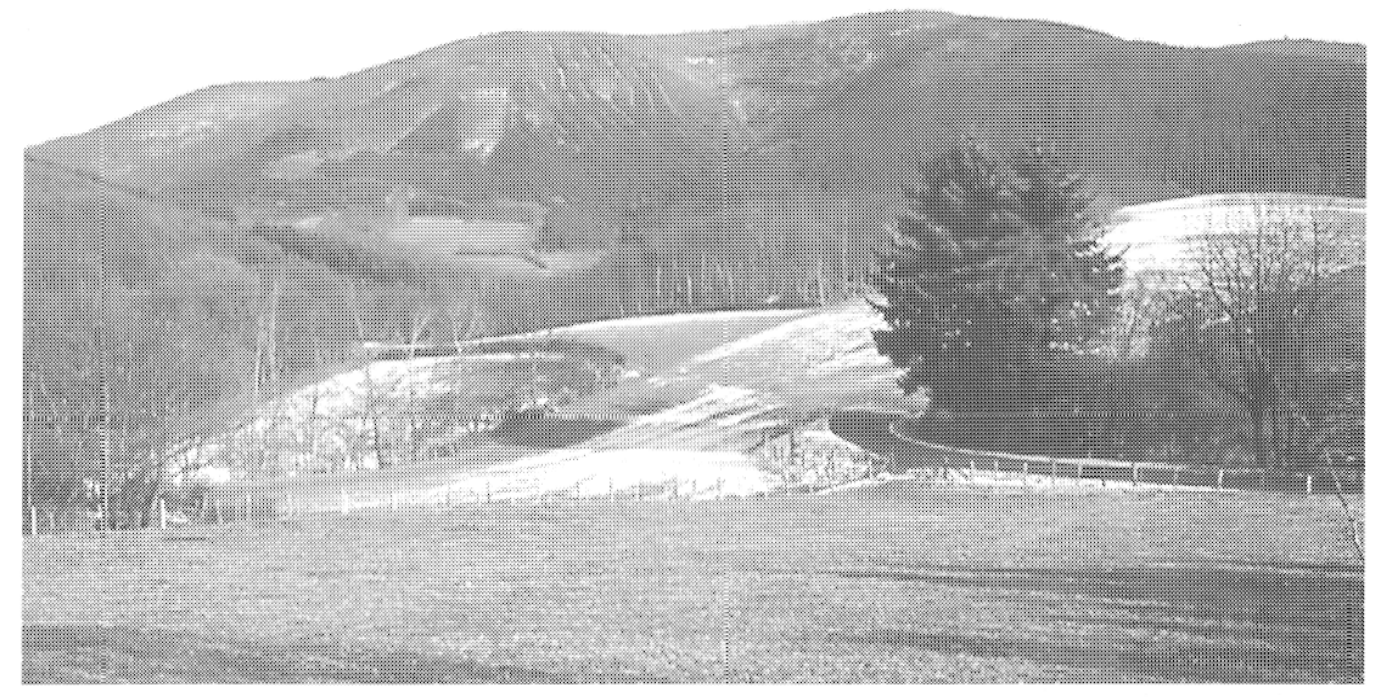

contextes naturels et humains. Dans ce but, le groupe de travail "Environnement et Prospection " a été mis en place dès 1986 ; il réunit des spécialistes venant d'horizons très différents travaillant sur le milieu naturel et son évolution au contact de l'homme, sur le site et dans ses environs immédiats (Buchsenschutz, Richard éds., 1996).

Dans un premier temps, les recherches privilégiant l'environnement actuel du site ont été menées de front avec celles qui touchaient à l'environnement ancien, accessibles seulement par la fouille. Si les premières sont presque toutes à terme aujourd'hui, les secondes sont encore en pleine évolution, continuellement alimentées par des informations nouvelles tirées des sondages et des prospections.

On pouvait penser, en débutant ces recherches, qu'il y avait de nombreux paradoxes dans l'occupation du Mont Beuvray à la fin de La Tène. Si le développement d'une ville à cet endroit s'explique assez bien par une situation privilégiée au point de rencontre des bassins de la Loire et de la Seine et de l'axe Saône-Rhône, l'installation sur cette montagne (fig. 3) défie apparemment toute logique à l'échelle de la microrégion si l'on se réfère aux conditions topographiques et climatiques : pluie, froid, brouillard, pentes, qui ne sont pas des condi- tions idéales pour une agglomération qui se veut urbaine.

Mais, si les conditions climatiques de cette région sont effectivement rudes, les études climatologiques montrent en fait que la température moyenne annuelle est somme toute assez élevée (de l'ordre de $8,5^{\circ} \mathrm{C}$ au sommet) et, surtout, que la saison froide est en moyenne peu accusée (Chabin, 1996). Ceci tend à prouver que « l'altitude idéale » pour l'homme, son habitat, ses jardins et ses vergers, se situe entre 500 et $600 \mathrm{~m}$ dans cette région. Des recherches plus globales sur l'évolution des paléoclimats (Richard, Magny éds., 1992) montrent aussi que l'époque où la ville de Bibracte a été créée correspondait à une période "plutôt favorable " du point de vue climatique.

Par ailleurs, les études sur la végétation moderne des environs du Mont Beuvray soulignent la grande diversité de la végétation potentielle (Perrier, 1996). Si cette richesse est aujourd'hui gommée par l'emprise anthropique, elle était un atout certain dans des temps plus reculés. Dans le même sens, l'analyse des macrorestes végétaux faite sur les principaux sites de l'oppidum montre la grande diversité des plantes cultivées, cueillies et stockées sur le site ( $c f$. infra, p. 63-65). Ces recherches, associées à la palynologie, l'anthracologie et la xylologie, 
révèlent qu'autour de l'oppidum l'espace est largement ouvert (Richard, 1996). Les cultures de céréales côtoient les prairies et les pâtures dans un paysage de bocage. À proximité des habitats sont cultivés, entre autres, des pois, des lentilles, des fèves. La nature fournit aussi une grande quantité de végétaux sauvages (baies, fruits, nombreuses plantes comestibles et médicinales). Mais, au delà de Bibracte et en dehors de la proximité des autres sites d'habitat, les Éduens (comme d'ailleurs la majorité des peuples gaulois) occupent un territoire encore largement forestier. Très diversifiées, les forêts de la Gaule tempérée sont dominées par le Chêne et le Hêtre associés à de nombreuses essences (Tilleul, Orme, Frêne, fruitiers sauvages et de très nombreux arbustes). La majorité de ces forêts est connue et exploitée pour le bois, les plantes comestibles et le gibier. Mais il reste de nombreux espaces difficilement pénétrables ; ces « forêts profondes» (Richard, 1995) ne seront conquises pour la plupart qu'à partir du Bas Moyen Âge.

Les occupants de l'oppidum de Bibracte ont également très bien su profiter de la complexité de l'environnement géologique. Le Mont Beuvray a fourni l'essentiel des volumes en terre et en pierre utilisés dans les constructions, ainsi que l'eau indispensable au développement de la communauté urbaine. À quelques heures de marche, les Éduens pouvaient se procurer des minerais de fer, des argiles et certains types de granites. Des roches à l'utilisation plus spécifique (certains éléments de construction ou les roches utilisées pour le broyage, la mouture, etc.) étaient également disponibles à des distances relativement proches ( $c f$. infra, p. 63).

Ce tableau ne doit pas pour autant masquer les réalités du lieu : le Morvan est un pays rude. Mais cette difficulté n'a semble-t-il pas toujours été répulsive pour l'homme. Depuis longtemps, probablement dès la fin du Néolithique comme le montrent les analyses palynologiques faites à la tourbière proche des sources de l'Yonne, l'homme s'est fixé dans cette région (Richard, 1996). Il laisse aussi son empreinte sur ce territoire. L'érosion profonde de certains sols ne peut être que d'origine anthropique (Veyret, Plassiard, 1996). La toponymie (Barral, 1996) et les nombreux sites repérés par la prospection (Goguey, 1996 ; Niaux, 1996) montrent que cette microrégion a toujours été largement peuplée et exploitée.

Il reste des lacunes dans notre connaissance de ce pays. Il faut par exemple mener à bien des études exhaus- tives sur les sols très complexes, en particulier à l'intérieur du site, et sur l'hydrologie. Des travaux prometteurs sont en cours sur les chemins anciens, sur la prospection systématique des pentes de l'oppidum, sur les meules; des essais d'intégration des données de la prospection dans un système d'information géographique s'orientent vers une analyse de l'évolution chronologique du paysage ; les résultats de l'étude des graines sont prometteurs pour restituer la physionomie des terroirs de l'époque, le calendrier des travaux agricoles, les modes de moisson et le stockage, l'utilisation des plantes cultivées.

Une étude régionale autour d'un site aussi important que Bibracte réunit tous les ingrédients pour éviter aussi bien les généralisations abusives que le côté anecdotique des monographies locales. À des textes historiques précis et importants répondent des vestiges archéologiques tangibles. Carrefour et refuge à la fois, le Morvan est un pivot interrégional en même temps qu'un conservatoire de pratiques archaïques. Ses cultures, à la limite des zones fertiles, réagissent vivement aux moindres variations climatiques ou économiques. Ici il est possible de combiner toutes les données, textuelles, archéologiques, environnementales, pour examiner l'histoire sous toutes ses facettes, sans en privilégier aucune.

O. B., H. R.

(Olivier Buchsenschutz, Hervé Richard)

\section{HISTOIRE DE L'OCGUPATION DU MONT BEUVRAY}

L'évolution de l'oppidum ne peut s'envisager que dans une approche diachronique de l'ensemble du site (fig. 4). Deux aspects inégalement étudiés pour l'instant sont en effet essentiels à la compréhension du rôle et de la place de l'oppidum dans la civilisation celtique. Qu'existait-il en ce lieu avant son occupation durable à La Tène finale? Peut-on envisager une installation ex nihilo, ou bien au contraire le choix des Éduens d'y installer leur principal oppidum s'inscrit-il dans une tradition beaucoup plus longue de fréquentation du site? De même, la romanisation entraîna-t-elle l'abandon total du site ? Comment et dans quel ordre s'opéra le transfert de la population de Bibracte à Augustodunum?

Pour les phases antérieures à La Tène moyenne, nous n'avons encore que peu d'éléments en dehors des résultats de prospections, de quelques témoins stratigraphiques ponctuellement mis en évidence et de mobilier 


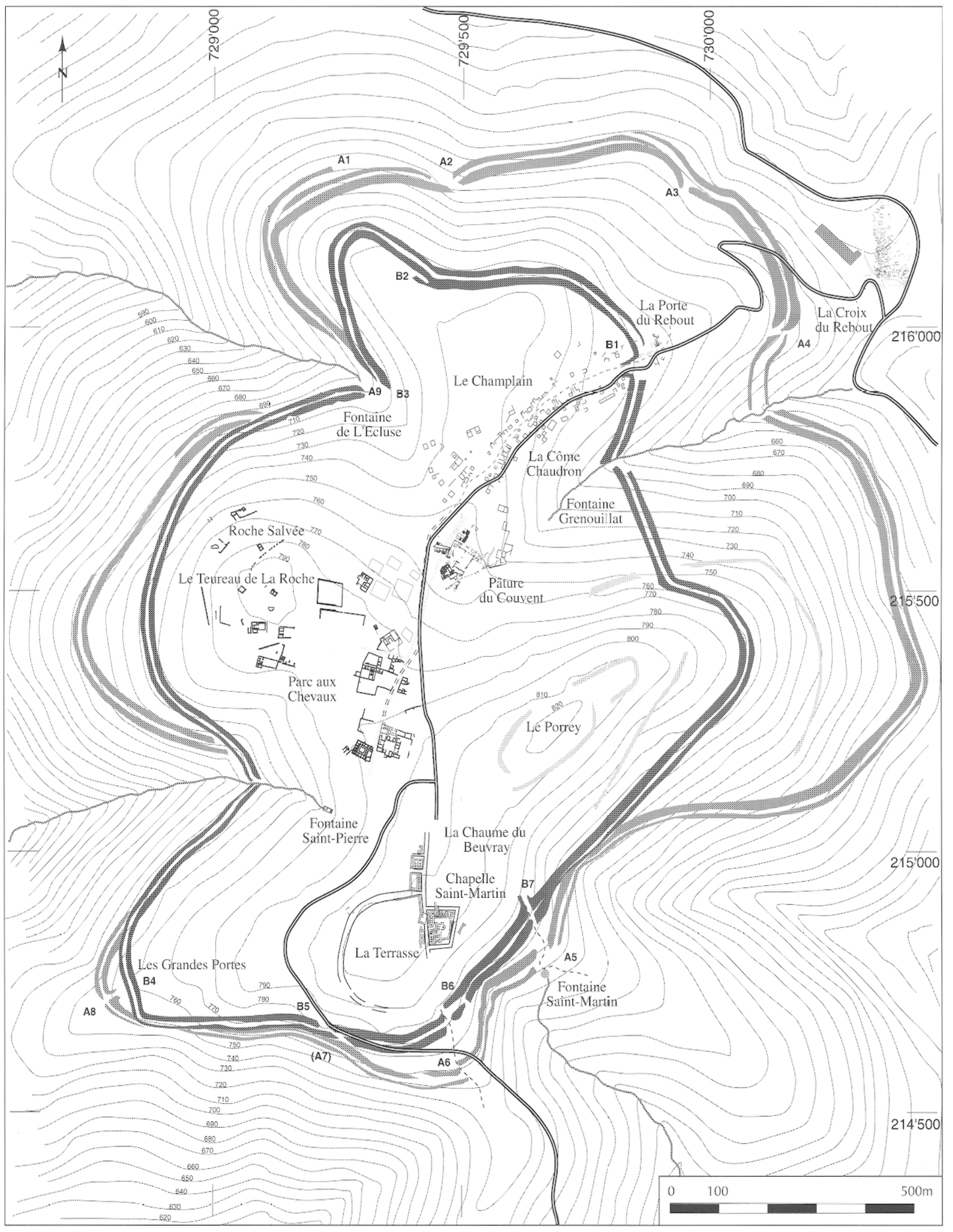

Fig. 4 - Carte archéologique et toponymie du Mont Beuvray. Sont notamment figurées les deux lignes de fortifications : en noir, la fortification intérieure " $B$ " reconnue par Bulliot; en gris, la fortification extérieure " $A$ » identifiée en 1986. 
provenant pour l'essentiel des fouilles anciennes, qui attend encore une réétude complète. Les priorités données à des zones densément occupées à l'époque gauloise et romaine expliquent sans doute en partie la pauvreté des résultats dans ce domaine.

La découverte de mobiliers très dispersés de l'Âge du Bronze et du Premier Âge du Fer sur la partie haute du massif et d'outillage néolithique sur l'ensemble des terrains étudiés est cependant la preuve d'une fréquentation ancienne. Des datations isotopiques obtenues sur des charbons de bois issus des couches profondes recouvertes par les remparts de La Tène finale, à la Porte du Rebout, ont livré trois dates qui suggèrent aussi une activité dès le Néolithique ${ }^{8}$.

Des données un peu plus conséquentes sont disponibles pour La Tène moyenne, quoique toujours modestes. Des mesures isotopiques et dendrochronologiques ${ }^{4}$ suggèrent ainsi que le talus de la plate-forme réservée de la Terrasse, située au sud de la montagne (fig. 18), a été installé au III ${ }^{\mathrm{e}}$ s. avant J.-C. Cette datation mérite toutefois d'être confirmée par d'autres mesures ou, mieux, par du mobilier archéologique. Il est vrai que c'est une hypothèse séduisante d'imaginer qu'un lieu de rencontre (cultuel ?) qui ne sera jamais bâti ( $c f$. infra, p. 31) est à l'origine de l'installation de l'oppidum. Quoi qu'il en soit, le mobilier protohistorique antérieur à La Tène D1 demeure très rare, tant dans les fouilles anciennes que dans les travaux de la dernière décennie (Gran-Aymerich, 1989) ${ }^{5}$.

3. Ces mesures d'activité du radiocarbone (L.y $5358: 7390 \pm 170 \mathrm{BP}$; Ly $5356: 6110 \pm 170 \mathrm{BP}$; Ly $5359: 5350 \pm 150 \mathrm{BP}$ ), obtenues sur des échantillons peu propices à l'expérience, ne permettent toutefois pas de déterminer une date précise, encore moins de dater la palissade à laquelle ils sont associés.

4. Mesure d'activité du radiocarbone Ly $5357: 2310 \pm 45 \mathrm{BP}$, soit entre 511 et 224 avant J.-C. en données calibrées. Hypothèse de datation de 241 avant J.-C., proposée sous toutes réserves, pour la fin d'une séquence longue de 75 années reconstituée à partir de 7 charbons de Hêtre et synchronisée avec les courbes régionales de référence du Chêne (rapport d'analyse du 15 septembre 1995, par G. Lambert et C. Lavier, UMR 9946 du CNRS).

5. On note ainsi l'absence totale des fibules de schéma La Tène II (ou de type plus ancien) reconnaissables parmi la collection de quelque 280 fibules recensées dans les fouilles anciennes (Guillaumet, 1984), sinon une possible fibule de schéma La Tène I en cours de fabrication (GranAymerich, 1989, fig. 1.3, p. 345). On peut encore signaler deux vases à piédestal de la fin de La Tène ancienne ou du début de La Tène moyenne, de provenance toutefois incertaine (ibid., fig. 5.1 et 5.2, p. 351), et seulement deux bracelets en verre, complets, appartenant à
On peut encore présumer de l'ancienneté du rempart " extérieur » mis en évidence dans les années 1980, puisque la topographie suggère qu'il est systématiquement recoupé par le rempart intérieur reconnu par les fouilles de Bulliot et les sondages récents de la Porte du Rebout. La superficie enclose par ce rempart externe long de $7 \mathrm{~km}$ est d'environ 200 ha. Il semble donc témoigner d'un état primitif de l'oppidum, plus étendu, dont la datation précise doit être une priorité des recherches à venir sur le site. De récentes prospections suggèrent aussi l'existence de vestiges de fortifications anciennes à l'intérieur de l'oppidum. La plus importante enserre l'ensemble de la montagne du Porrey, soit 5 ha, et demeure non datée, malgré des sondages effectués en ce sens (Almagro-Gorbea, Gran-Aymerich, 1991, p. 317$331)$.

L'évolution du site est aussi tributaire des cheminements qui le traversent. Deux axes principaux peuvent être discernés. Le premier, nord-sud, passe par les sources de l'Yonne - lieu d'un sanctuaire gallo-romain de source -, le col de l'Échenault, la Roche de la Wivre, le Porrey, la Porte Saint-Martin et se poursuit vers la vallée de l'Arroux. Le second, est-ouest, est celui encore utilisé de nos jours, de la Porte du Rebout aux Grandes Portes de Nevers. On peut supposer - mais la preuve archéologique reste à faire - que le premier, qui correspond à un itinéraire « naturel " suivant la principale ligne de crête du Morvan depuis Avallon, au nord, jusqu'au mont Dardon, au sud, est plus ancien que l'autre, qui met en communication Bibracte avec Autun.

L'oppidum est sans aucun doute quasi totalement déserté par ses habitants en l'espace de quelques décennies, tout au plus, environ un demi-siècle après la conquête. Les points qui ont livré des traces d'une fréquentation régulière à l'époque romaine n'ont plus de vocation résidentielle; il s'agit de lieux publics : sanctuaire de la Chaume ( $c f$. infra, p. 31) et Fontaine SaintPierre (cf. infra, p. 34). Leur usage se prolonge aux époques médiévale et moderne, tandis que la montagne

des types de La Tène C2 (l'un de la série lla de Gebhardt, l'autre violet, du groupe Heavernick 6C), issus des fouilles de 1870 à la Côme Chaudron (objets inédits et apparemment perdus, illustrés dans les Albums de Bulliot). Vient encore moduler ce constat la présence sporadique, dans des contextes plus récents, d'amphores vinaires gréco-italiques dont la production ne paraît pas se prolonger au-delà du milieu du $\mathrm{II}^{\mathrm{e}}$ s. avant J.-C. (cf. p. 78). 
accueille pendant quelques siècles un petit établissement monastique, d'abord une grange bénédictine (non localisée), puis un couvent franciscain, $\mathrm{du} \mathrm{XV}^{\mathrm{e}}$ au XVII ${ }^{\mathrm{e}} \mathrm{s}$. Des foires saisonnières s'y tiennent encore au XIX ${ }^{\mathrm{c}} \mathrm{s}$., sans que l'on puisse affirmer qu'il s'agit du maintien depuis l'Antiquité du rôle de marché que jouait l'oppidum.

Dans l'état actuel de nos connaissances, l'occupation maximale du site apparaît donc bien se situer au I $^{\text {er }} \mathbf{s}$. avant J.-C. La complexité de la topographie de l'oppidum du temps de César et d'Auguste est, certes, liée au relief, mais sans doute aussi aux différents axes de circulation qui le traversent et à un passé plus lointain, dont on commence seulement à soupçonner le poids. Quelques indices suggèrent en effet que l'agglomération du $\mathrm{I}^{\mathrm{cr}} \mathrm{s}$. avant J.-C. trouve son origine à une époque plus ancienne, dont les vestiges les plus plausibles révélés par les travaux récents sont le rempart extérieur et l'aménagement de la Terrasse. La poursuite de leur reconnaissance est essentielle si l'on veut mieux cerner les origines du phénomène général de développement des vastes centres fortifiés de l'Europe tempérée que sont les oppida.

J.-P. G., V. G. (Jean-Paul Guillaumet, Vincent Guichard)

\section{APRÈS BIBRACTE, ACTIVITÉS RELIGIEUSES ET SURVIVANCE D'UNE FRÉQUENTATION SAISONNIÈRE}

\section{La Chapelle Saint-Martin}

La reprise des fouilles de la Chapelle Saint-Martin, sur le sommet de la Chaume, répondait à deux objectifs : rechercher les vestiges d'un hypothétique sanctuaire antérieur au temple gallo-romain mis en évidence par Bulliot, et préciser la chronologie relative et le plan des différents états du sanctuaire chrétien qui a succédé au temple antique (fig. 5). Sur le premier point, les résultats sont limités ( $c f$. infra, p. 31). Sur le second, en revanche, la fouille apporte des données substantielles (Beck et al., 1988). Si l'existence d'un fanum romano-celtique classique n'était plus discutée, l'interprétation des structures postérieures continuait en effet à soulever des questions (fig. 6). La destruction par les fouilles antérieures à 1984 de l'essentiel des liens stratigraphiques rend toujours difficile toute chronologie fine du site (Beck et al., 1987). Référence faite aux autres monuments du même type fouillés depuis, il apparaît que rien de probant ne permet de rattacher une transformation de celui-ci à l'époque paléochrétienne. C'est donc plus vraisemblablement au $\mathrm{X}^{\mathrm{e}} \mathrm{s}$. et à l'époque romane qu'il convient de trouver des parallèles aux modifications des bâtiments que justifient plus sérieusement l'histoire du site et ses liens avec les abbayes autunoises. L'étude du mobilier céramique conforte ce point de vue : elle démontre une permanence de l'occupation antique jusqu'au milieu du $\mathrm{V}^{\mathrm{k}} \mathrm{s}$., tandis que les époques postérieures au IX ${ }^{\mathrm{c}} \mathrm{s}$. sont ensuite à nouveau bien représentées. On peut donc en déduire qu'il n'y a pas nécessairement eu de transformation précoce de l'édifice gallo-romain, même s'il y a eu continuité de son utilisation. Ainsi, en milieu rural, en dépit d'une christianisation dès la fin du IV e s., on voit subsister très tard et sur plus de trois siècles des constructions d'origine païenne. On ne peut ici mesurer le degré de christianisation et encore moins le mode d'utilisation de ces constructions, mais simplement constater que l'on continue à les entretenir sans les détruire. Comme le Mont Beuvray, d'autres sites de hauteur de la région (Suin, Sainte-Colombe, Mont Dardon, La Certenue) offrent des faciès variés qui montrent des hiatus importants entre une origine précoce et leur développement dans le Haut Moyen Âge.

\section{Le couvent des Cordeliers du Mont Beuvray : XIV $^{\mathrm{e}}$-XVIII ${ }^{\mathrm{e}} \mathrm{s}$.}

Un curieux épisode marque l'apparition du couvent franciscain du Mont Beuvray dans l'histoire : en 1424, frère Étienne Charlot, moine cordelier du Beuvray, est arrêté au château de Larochemillay sous l'inculpation d'espionnage pour le compte d'Odette de Champdivers, ancienne favorite du roi de France Charles VI (archives départementales de la Côte-d'Or, B 11290). Pour être marquant, l'événement, lié à la guerre de Cent Ans, reste isolé et la destinée de l'établissement fut généralement plus discrète.

Le couvent est fondé quelques années auparavant sous la protection et avec l'aide financière des barons de Larochemillay, sur des terres exploitées au moins depuis le $\mathrm{XI}^{\mathrm{e}}$ s. par les bénédictins d'Autun. Il abrite une communauté de moines observants, c'est-à-dire des moines franciscains engagés dans un mouvement de réforme caractérisé par la stricte observance de la règle édictée deux siècles auparavant par saint François d'Assise, qui se 


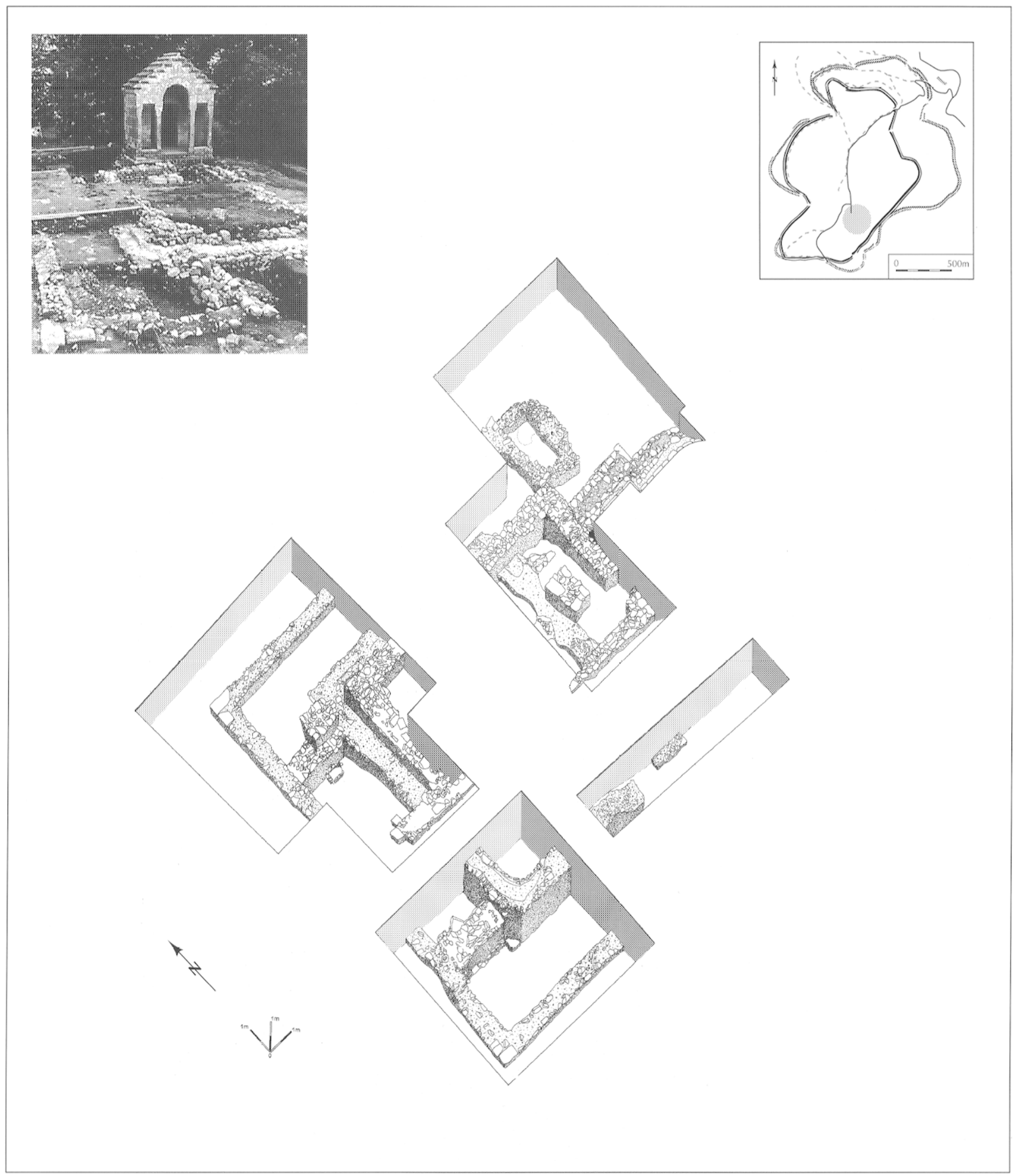

Fig. 5 - Sanctuaire situé sous la Chapelle Saint-Martin, vue générale de la fouille et vue axonométrique des différents états de construction. 


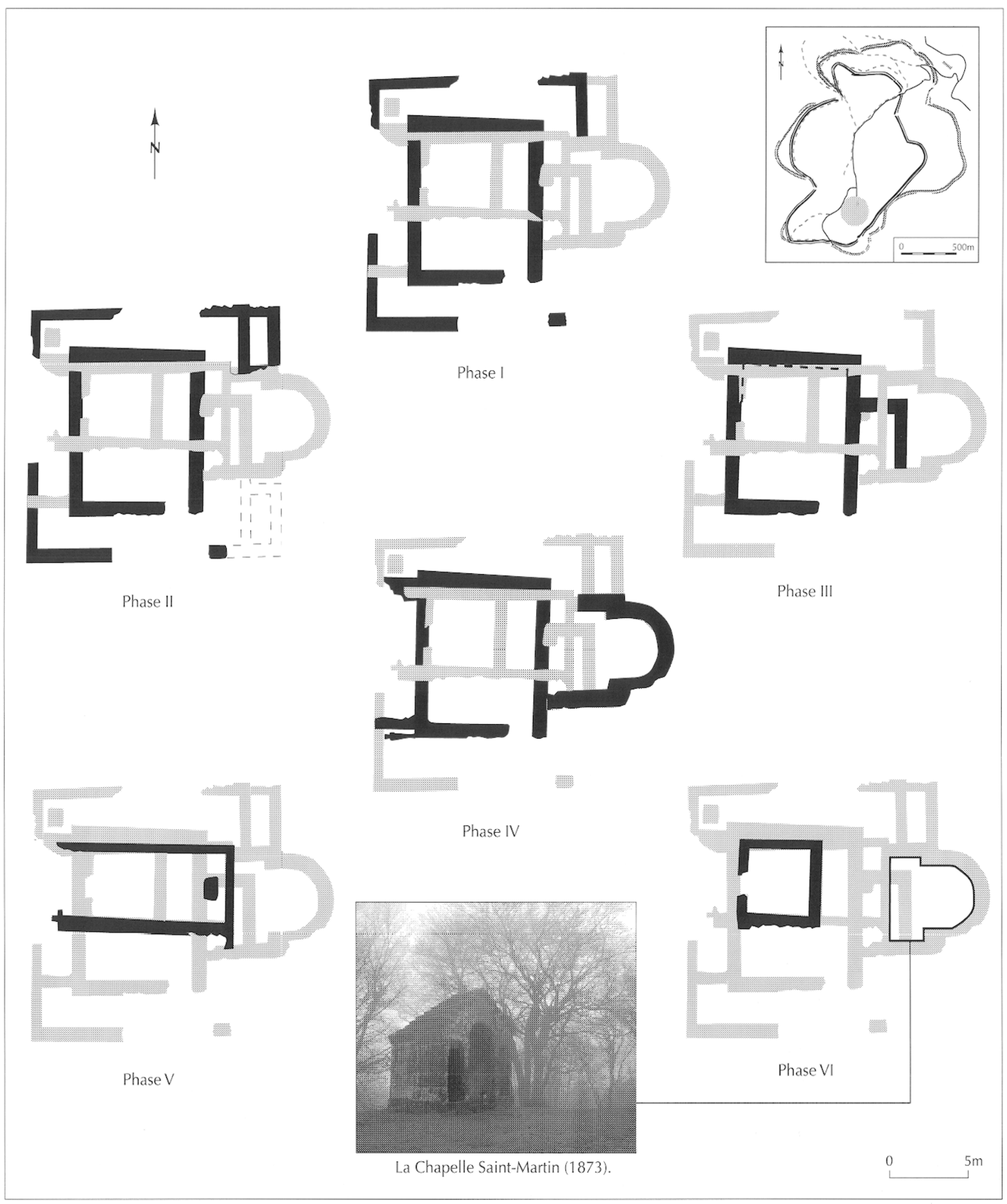

Fig. 6 - Évolution du sanctuaire situé sous la Chapelle Saint-Martin : phases I et II, fanum, époque gallo romaine ; phase III, chapelle, VII'-VIII s. ; phase IV, chapelle, $X^{e}$ s. ; phase V, chapelle, XIII ${ }^{e}$ s. ; phase VI, oratoire, XVII s. 
rallièrent très tôt à la réforme de saint Colette (RF, 1994, p. 173).

Dans ce lieu reculé et inhabité depuis la disparition de la Bibracte antique, animé toutefois chaque année par des foires et des pèlerinages à la Chapelle Saint-Martin, les frères vivent des revenus de leurs terres, des aumônes et de leurs prédications. C'est ainsi que le chancelier de Bourgogne, Nicolas Rolin, les gratifia d'un franc en 1446 et que dix ans plus tard, « frère Pierre Chambon, gardien du Couvent de Beuvray, recevait dix aulnes de bure pour avoir prononcé vingt et un sermons à Nevers. » (archives municipales de Nevers, CC 52).

En 1538, alors que la communauté compte cinq religieux, les bâtiments sont ruinés par un incendie, mais ils sont bientôt restaurés : frère Jean de Marry demande à sa famille de disposer de son patrimoine pour la réparation et l'entretien du couvent [...] « lequel depuis peu de temps avoit esté du tout par feu bruslé, desmoly et n'estoit reffaict " (Beaudiau, 1865, I, p. 358).

$\mathrm{Au}$ début des années 1570, nouvelle destruction, séquelle locale des guerres de Religion, et nouvelle restauration (Gueneau de Vandenesse, 1875) ; pendant près d'un siècle encore, les moines exercent leur sacerdoce dans les paroisses voisines, notamment à Saint-Légersous-Beuvray, où on les trouve cités dans les registres paroissiaux (Saint-Léger-sous-Beuvray, Saône-et-Loire ; registre paroissial, livre des baptêmes, 1641).

L'abandon et le repli sur le couvent franciscain d'Autun sont effectifs à la fin du XVII ${ }^{e}$ s. : l'établissement est réputé abandonné en 1699 (archives départementales de la Côte-d'Or, 49 H 972) et, en 1737, Étienne Marceau, marchand de Glux, se porte acquéreur de "l'ancienne place où, autrefois étoit basty le couvent desdits pères cordeliers sur la montagne de Beuvray entièrement démoly $[\ldots]$ masure qui y peut estre qui n'est qu'une vieille cave, la pâture, arbres qui y peuvent être " (archives départementales de Saône-et-Loire, série H, supplément; cordeliers d'Autun, liasse 4, cordeliers de Beuvray).

Sur les cartes et le terrain, la toponymie et la topographie signalaient parfaitement l'emplacement de l'établissement. Au lieu-dit la Pâture du Couvent, un puissant pierrier de près de $1000 \mathrm{~m}^{2}$ de superficie et $3 \mathrm{~m}$ de hauteur, entouré d'une vaste terrasse quadrangulaire délimitée par un muret ponctuellement visible encore, était aisément repérable dans le paysage. Le plan, dressé en 1989, en montra l'organisation : six dépressions délimi- tées par des couronnes pierreuses évoquaient des locaux jointifs enserrant sur quatre côtés un espace vide, le cloître sans doute.

Les fouilles archéologiques menées depuis 1989 ont découvert un ensemble fort complexe, qui avait subi non seulement deux incendies, correspondant sans doute aux deux épisodes évoqués par les textes, mais aussi de nombreuses restructurations du XV' au XVII's. Il s'agit du couvent et de ses annexes de production, qui se superposent à quelques constructions plus anciennes, médiévales sans doute, mais difficilement datables et identifiables, elles-mêmes faisant suite à de puissant remblais masquant un quartier de la cité antique (fig. 7).

Au XVe et au XVI ${ }^{e}$ s., c'est un beau monument d'environ $1000 \mathrm{~m}^{2}$ de superficie, qui ne se limite pas aux aménagements conventuels classiques distribués autour d'un cloitre, mais développe fortement en excroissance vers le nord un pôle d'activités domestiques et de production.

Au sud, l'espace religieux se définit par une grande église $(\mathrm{K} / \mathrm{M})$ de plan rectangulaire s'achevant à l'est par un mur absidial semi-circulaire. Le chour, desservi par deux annexes liturgiques ( $L$ et $N$ ), est séparé de la nef par une barrière. Éclairé par de grandes baies de style gothique tardif, notamment au chevet, cet édifice est au moins partiellement voûté : une clé d'ogive, trouvée en remploi, présente un écusson frappé aux armes de Nicolas Rolin. Ses parements internes sont masqués par un enduit de chaux blanche localement peint. Sur son flanc nord, le cloître à galeries de $14 \mathrm{~m}$ de côté accueille des inhumations en son préau (I et J).

Les locaux de l'aile est présentent trois niveaux d'utilisation : des caves voûtées (en $G$ et $R$ ), un rez-de-chaussée aux grandes pièces dallées et un étage (dortoir ?) desservi par un escalier droit aménagé dans le local $\mathrm{P}$ à la jonction de l'église et de la galerie du cloître.

$\mathrm{Au}$ nord, les installations domestiques et de production ferment au nord et à l'est une cour délimitée à l'ouest par le mur d'enclos. L'aile septentrionale du cloître accueille notamment une cuisine pavée (B), munie d'une cheminée et d'un dispositif d'évacuation des déchets (D), ainsi qu'un ensemble de locaux de service aux sols dallés ( $U$ et $Y$ puis $Q$ ), disposés en excroissance et intégrant les escaliers d'accès aux caves ( $T$ et $R$ ). $\grave{A}$ environ $20 \mathrm{~m}$, dans l'angle nord-ouest de l'enclos conventuel, six locaux jointifs s'étendent en ligne sur $27 \mathrm{~m}$ de longueur. Avec une maison d'habitation signalée par un foyer (IV), une grange ouvrant par un grand 


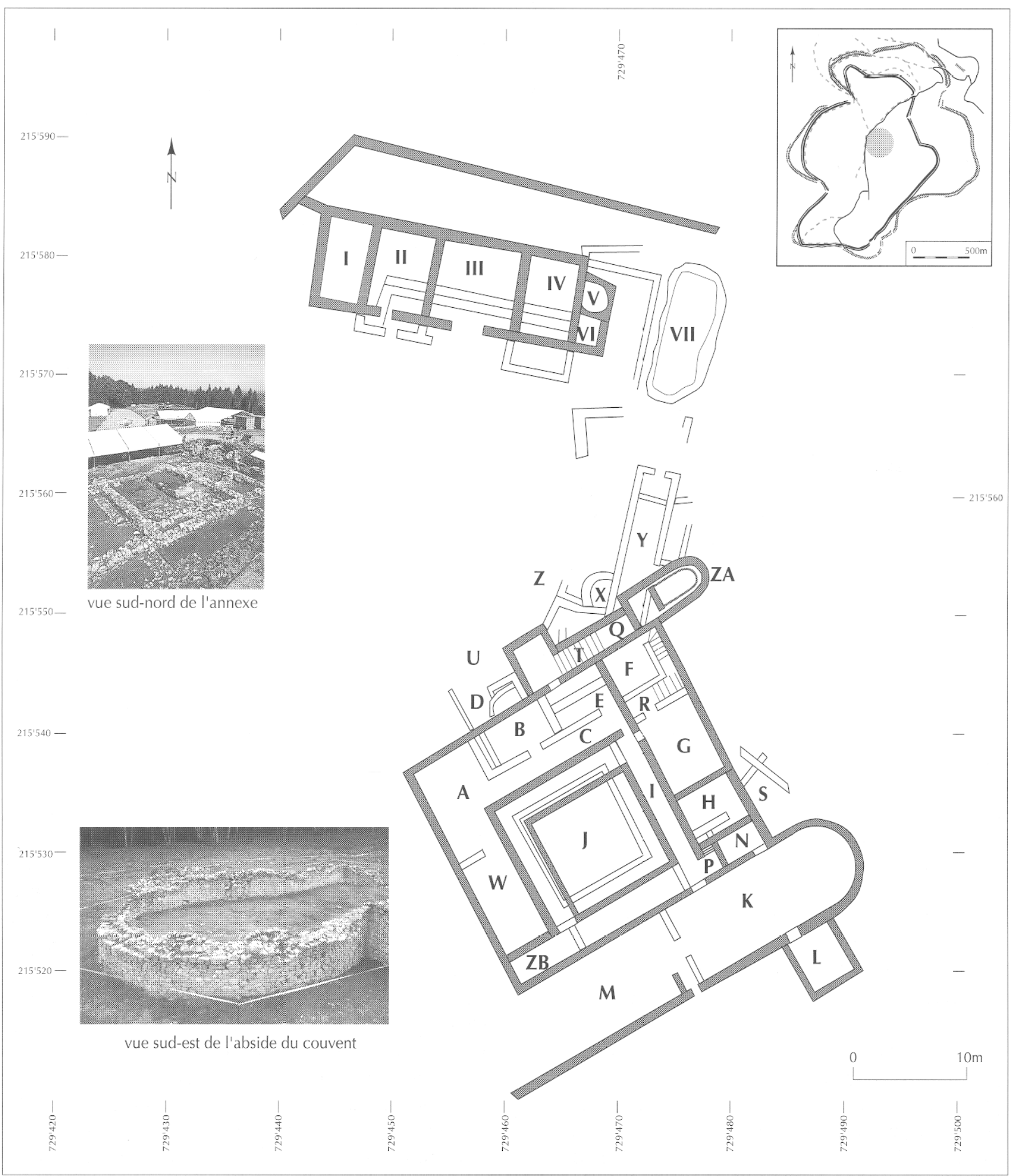

Fig. 7 - Plan d'ensemble du couvent franciscain et de ses annexes. 
portail (III), un fournil (V et VI) et deux autres locaux jointifs (I et II), ils constituent les annexes agricoles.

Sous le couvent et ses annexes enfin, de nombreuses canalisations enterrées, constamment remaniées, prolongent des drains courant sous les sols : l'eau de la fontaine, située immédiatement en amont de la chapelle et alimentant le ruisseau de la Côme Chaudron, est ainsi captée pour assainir le terrain, fournir la communauté, évacuer ensuite les déchets domestiques de deux latrines (D et $\mathrm{ZA}$ ) vers une fosse septique flanquant à l'est les annexes d'exploitation (VII).

Plusieurs dizaines de monnaies des XIV"e-XVII ${ }^{\mathrm{e}}$ s. et un mobilier domestique abondant et diversifié, céramique, métallique, vitreux et ostéologique accompagnent les structures. Leur analyse, en cours, permettra d'identifier mieux encore les fonctions des différents locaux et d'éclairer l'environnement matériel de cette communauté monastique.

L'enquête sur le terrain reste aujourd'hui inachevée : une importante campagne de fouille est encore nécessaire pour terminer l'exploration de l'église conventuelle et des extérieurs, et pour révéler les aménagements de la fontaine; parallèlement, une prospection autour du Mont Beuvray doit apporter des éléments de comparaison sur lcs tcchniques ct les matériaux de construction et sur l'organisation spatiale des aménagements.

Cette recherche, intégrée aux travaux des autres équipes du Centre archéologique européen, livre des éléments riches et originaux tant sur l'histoire de l'ordre franciscain que sur celle du Mont Beuvray; la conservation et la restauration des vestiges, sans gêner l'étude des structures antiques sous-jacentes, devraient en assurer la lisibilité culturelle.

P. B., W. B., C. C., K. G., J. L., B. St-J.-V., C. S. (Patrice Beck, Walter Berry, Christine Canat, Katherine Gruel, Josef Laszlovsky, Benjamin Saint-Jean-Vitus, Christian Sapin)

\section{L'ORGANISATION SPATIALE DE L'OPPIDUM}

Les vestiges remontant à La Tène $\mathrm{D}$ et à l'époque augustéenne atteignent une ampleur incomparable avec les faibles traces des périodes précédentes. Ils se caractérisent par la mise en place d'une voirie, la maîtrise de la circulation des eaux, la construction de fortifications, la délimitation d'espaces publics ou religieux qui structurent un habitat étendu sur une surface considérable.

\section{LES FORTIFICATIONS}

La fortification du Mont Beuvray, bien que masquée aujourd'hui sous les arbres et sous ses propres éboulis, reste un des monuments les plus spectaculaires que nous aient légués les populations de l'Âge du Fer (fig. 8). La hauteur du rempart, précédé d'un fossé et d'une contrescarpe qui dessinent aujourd'hui à son pied une large terrasse, le classe déjà parmi les fortifications les mieux conservées du monde celtique. Mais c'est surtout la continuité du tracé, ininterrompu sur $5 \mathrm{~km}$, qui force l'attention. Bulliot (1899, I, p. 1-95) avait déjà reconnu cette volonté de dresser une limite artificielle entre l'espace urbain et la campagne, soulignée encore par des portes monumentales. Déchelette avait fait les comparaisons qui s'imposaient avec Manching (Allemagne) et Stradonice (République tchèque). L'archéologie confirme ici le témoignage des textes qui attribuent à Bibracte le rôle de capitale d'une des plus puissantes tribus de la Gaule.

La reconnaissance d'une seconde fortification, englobant celle de Bulliot, est l'un des apports les plus neufs des campagnes de fouilles et de prospection récentes (fig. 4). L'acquisition des pentes de la montagne par l'État et leur nettoyage consécutif ont permis de découvrir ce nouveau rempart qui se confondait jusque-là avec les multiples chemins, antiques ou modernes, se croisant et se recoupant jusqu'à la base du massif. F. Schubert a parcouru en tous sens ce territoire et effectué des relevés minutieux pour chaque ondulation du terrain. Il a pu avancer de solides hypothèses qui seront à confirmer par des fouilles dans les années à venir. Ce rempart " externe » entoure une surface de près de 200 ha, équivalente à celle qu'occupe la ville d'Autun; toutes les observations sur les microreliefs semblent indiquer qu'il est antérieur au rempart " interne ". Cette séquence chronologique, si elle est confirmée par des sondages, nous amènera à re considérer l'évolution de l'oppidum, et surtout l'écart entre le programme initial et l'étendue effective de l'agglomération.

Les prospections géophysiques sur les clous du murus gallicus, conduites par A. Hesse et K. Aitchison, ont permis jusqu'à présent de repérer cinq fiches de fer sur le rempart externe. C'est bien sûr un indice insuffisant pour en tirer des conclusions : il faudra attendre le résultat des fouilles entamées par l'université de Vienne 